\title{
Conservative CIP Transport in Meteorological Models*
}

\author{
Xindong PENG $^{* *}$, Feng $\mathrm{XIAO}^{* * *}$, Keiko TAKAHASHI ${ }^{* * * *}$ and Takashi YABE ${ }^{\dagger}$
}

In order to improve the model representation, we have implemented the CIP-CSLR in meteorological models. Real-case simulations and idealized tests were carried out on the Earth Simulator with atmospheric general circulation models that only the watervapor and liquid water were transported with the CIP-CSLR method. Numerical experiments show that the CIP-CSLR scheme substantially improved the model outputs for both pure advection tests and the long-term climate simulations. Reasonable tropical precipitation is shown with the CIP-CSLR scheme, which is largely improved in comparison with the original spectral method. Using the CIP-CSLR on a new grid (Yin-Yang) system, we also achieved conservative and more accurate results of passive tracer advection with relatively fewer grid points compared with the latitude-longitude grid. Without polar singularity, the splitting procedure of CIP-CSLR achieves promising transport in spherical geometry even if large Courant number is specified.

Key Words: CIP, Semi-Lagrangian Scheme, Conservation, Yin-Yang Grid, Meteorological Model

\section{Introduction}

Computation of advection transport plays a key role in atmospheric/meteorological models. The quality of the numerical solutions of the advection processes substantially affects the outputs of the whole numerical model. When applied to the numerical advection of the air-borne substances, such as chemical species or water substances, a numerical scheme is usually required to be conservative, positive-definitive, less diffusive and computationally efficient.

Because of the rapid development of computer hardware, an increasing number of powerful computational facilities are getting installed at many institutes over the world, which allows even a global model to run with

* Received 19th March, 2004 (No. 04-4090)

** Earth Simulator Center, JAMSTEC, 3173-25 Showamachi, Kanazawa-ku, Yokohama 236-0001 Japan. E-mail:peng@jamstec.go.jp

*** Department of Energy Sciences, Tokyo Institute of Technology, 4259 Nagatsuta-cho, Midori-ku, Yokohama 2268502, Japan. E-mail: xiao@es.titech.ac.jp

**** Earth Simulator Center, JAMSTEC, 3173-25 Showamachi, Kanazawa-ku, Yokohama 236-0001 Japan.

E-mail: takahasi@jamstec.go.jp

$\dagger$ Department of Mechanical Engineering and Science, Tokyo Institute of Technology, 2-12-1 O-okayama, Meguroku, Tokyo 152-8550, Japan.

E-mail: yabe@mech.titech.ac.jp very high spatial resolutions. One of the most noteworthy supercomputers in the world is the Earth Simulator which has a peak performance of 40 TFLOPS for its total 640 nodes (5 120 parallel-vector processors). Its processing capability not only won itself the "SC2002 Gordon Bell Award", but also realized for the first time the global meso-scale simulation (general circulation model for global atmosphere with $10-\mathrm{km}$ grid spacing). With a refined grid, the detail structures that were considered to be of sub-grid scale in the past become directly resolvable. As a consequence, large gradients or discontinuities are more likely to appear in the numerical solutions, especially those associated with squall line, front, hurricane and other violent system. So, high resolution simulations require more faithful numerical advection that is capable of capturing not only the smooth solution, but also the large gradients and discontinuities. For this purpose, the so called high resolution schemes originally developed for aerodynamics, such as the Total Variation Diminishing (TVD) $^{(1)}$, the Monotonic Upstream-centered Scheme for Conservation Lows (MUSCL) ${ }^{(2)}$, the Piecewise Parabolic Method (PPM) ${ }^{(3)}$ and the constrained interpolation profile $(\mathrm{CIP})^{(4),(5)}$, have been used for the tracer transport in meteorological models. For further improving the numerical simulations of atmospheric and climatic dynamics, advection schemes with higher quality are still demanding.

We have recently devised a practical advection scheme, namely CIP-CSLR ${ }^{(6)}$ (Constrained Interpola- 
tion Profile Conservative Semi-Lagrangian with Rational Function) based on the $\operatorname{CIP}^{(4)}$ concept. Different from the conventional high-resolution scheme, the CIP-CSLR uses both the cell-integrated average and the cell interface value as the prognostic variables and predicts them simultaneously according to governing equations. It is proved by both theoretical analysis and numerical experiments that the CIP-CSLR is conservative, dispersion-accurate, oscillation-free, less diffusive and computationally efficient. As an advection scheme, CIP-CSLR is expected to be practical and adequately accurate in meteorological and climatic applications.

In this paper, we introduce some of our recent studies to implement the CIP-CSLR scheme in the existing model developed for the Earth Simulator, the AFES (Atmospheric general circulation model For the Earth Simulator) $\operatorname{model}^{(7)}$ and a new type of Chimera grid system, called "Yin-Yang" grid. When applied to a spherical geometry, some extra difficulties come out, for example, the polar singularity in the latitude-longitude grid of the AFES and the conservative interpolation on the "Yin-Yang" grid.

In section 2, the CIP-CSLR scheme is briefly introduced. The implementation of the scheme to the latitudelongitude coordinate and the numerical experiments with AFES are given in section 3. The numerical treatments that conserve the advection and the application of CIPCSLR with idealized advection tests are presented in section 4 , and the paper ends with some conclusion remarks in section 5 .

\section{Brief Description of the CIP-CSLR}

The CIP-CSLR scheme ${ }^{(6)}$ is developed to solve the following continuity equation,

$$
\frac{\partial q}{\partial t}=-\frac{\partial u q}{\partial x}
$$

with $q$ being the transported quantity and $u$ the velocity.

Different from conventional conservative finite difference methods or finite volume methods, the CIP-CSLR scheme treats both the cell-integrated average and the cellinterface value as the model variables which are memorized and carried forward in time. Our numerical experiments and theoretical analysis show that using both the cell-integrated average and the cell-interface value leads to a much more accurate numerical dispersion than any other conventional scheme that uses only either the cellintegrated average or the cell-interface value as the single variable for the reconstructions. In the CIP-CSLR, the cell-interface value is updated by a semi-Lagrangian remapping, while the cell-integrated average is computed through a formulation of flux-form. Thus, the latter is exactly conserved.

Rational functions are used for the interpolation of reconstructions in CIP-CSLR. As discussed in Refs. (6) and (8), the rational function preserves the convexity of the transported data and naturally results in oscillation-free numerical solutions even in the presence of large gradient or discontinuity. Moreover, the CIP-CSLR appears to be more computationally efficient than other conventional schemes of the same numerical accuracy.

Concerning the multi-dimensional implementation, a simple but adequately accurate formulation was proposed in Ref.(6), where a splitting based on the 1D scheme was employed. Similar to any other conservative highresolution scheme, the multi-dimensional extension of the CIP-CSLR using dimensional splitting still appears to be computationally efficient, and can be straightforwardly incorporated with the Strang's time integration ${ }^{(9)}$ and the divergence correction ${ }^{(10),(11)}$.

\section{An Application to Tracer Transport in an AGCM}

In a spectral model, we must get rid of the difficulty of dealing with nonlinear advection term, because the computation is very expensive for the multiplication of two spectral series. In the original AFES, advection is computed on Gaussian grid points with central differencing method, and then transformed to spectral space, so as to avoid massive computation of the nonlinear terms with spectral representation. The numerical oscillation of the spectral method and central differencing scheme in the original model is evident, which shows as a problem in tracer transport. The resulting negative watervapor is artificially filled by extortion from its neighbor gridpoints. An alternative solution is the application of semi-Lagrangian scheme such as ${ }^{(12)-(14)}$ to compute advection on grid, and it tends to be widely applied to general circulation models for high-resolution simulation. In this way, the computation of nonlinear advection term becomes much accurate and economic.

We have incorporated the CIP-CSLR scheme to AFES model. When implementing the CIP-CSLR to the AFES, we need to solve the following transport equation in spherical geometry,

$$
\begin{aligned}
\frac{\partial q}{\partial t} & =-\frac{\partial}{\partial \lambda}\left[\frac{U}{a\left(1-\mu^{2}\right)} q\right]-\frac{\partial}{\partial \mu}\left[\frac{V}{a} q\right]-\frac{\partial \dot{\sigma} q}{\partial \sigma} \\
& +q\left[\frac{1}{a\left(1-\mu^{2}\right)} \frac{\partial U}{\partial \lambda}+\frac{1}{a} \frac{\partial V}{\partial \mu}+\frac{\partial \dot{\sigma}}{\partial \sigma}\right] ;
\end{aligned}
$$

where $q$ is a tracer variable (e.g. mixing ratio of water vapor), $\mu=\sin \varphi, U=u \cos \varphi, V=v \cos \varphi$ and $\varphi, \lambda$ the latitude and longitude, respectively. The $u, v$ denotes the zonal and meridional wind. $\sigma$ is the vertical terrain-following coordinate, and $\dot{\sigma}$ the vertical velocity. $a$ is the radius of the Earth. It is worthy noting that same as that in the original AFES, the tracer quantity $q$ in Ref. (2) is the mixing ratio instead of the mass.

As discussed in Ref. (10), a correction to the numerical flux in terms of velocity divergence is needed in each direction for a splitting scheme. Thus, the three dimensional computation is conducted by solving the following 

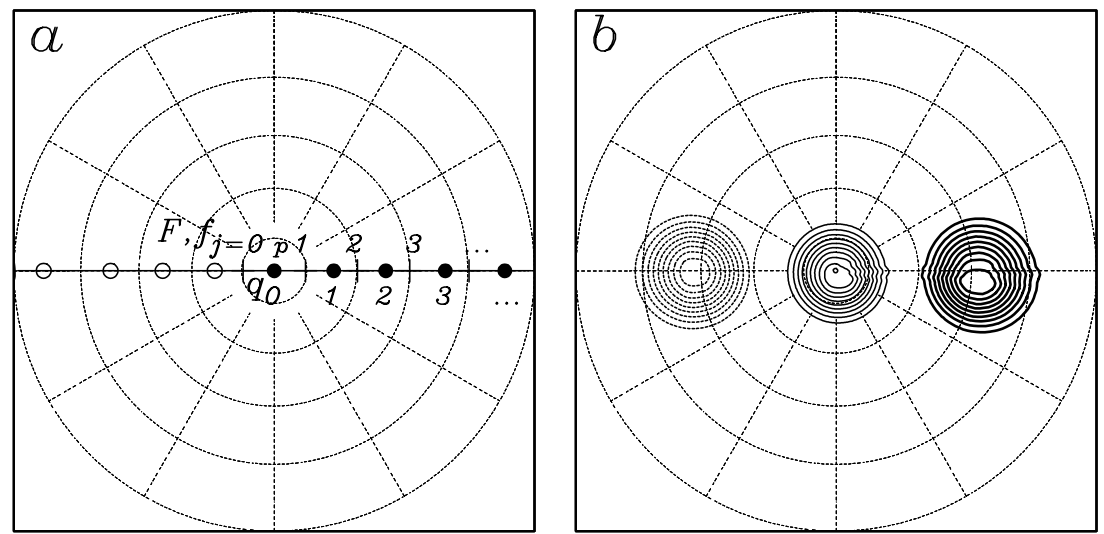

Fig. 1 (a) Schematic illustration of meridional advection in polar region. Closed circles show cell-integrated points at longitude $\lambda$ and open circles the cell-integrated points at $\lambda+\pi$, the opposite side. Cross points are the interfaces. $p$ is the South Pole $(j=0)$. (b) Advection of a cosine bell with the CIP-CSLR for $\alpha=\pi / 2$ in Williamson test 1 . The dashed and thick solid lines show the states before and after its going across the pole, respectively; and the thin solid lines show the advection over the pole.

equations where the last terms on the right-hand sides effectively work as the divergence correction.

$$
\left\{\begin{array}{l}
\frac{\partial q}{\partial t}=-\frac{\partial}{\partial \lambda}\left[\frac{U}{a\left(1-\mu^{2}\right)} q\right]+q\left[\frac{1}{a\left(1-\mu^{2}\right)} \frac{\partial U}{\partial \lambda}\right], \\
\frac{\partial q}{\partial t}=-\frac{\partial}{\partial \mu}\left[\frac{V}{a} q\right]+q\left[\frac{1}{a} \frac{\partial V}{\partial \mu}\right] \\
\frac{\partial q}{\partial t}=-\frac{\partial \dot{\sigma} q}{\partial \sigma}+q\left[\frac{\partial \dot{\sigma}}{\partial \sigma}\right] .
\end{array}\right.
$$

Because of the existence of polar singularity in latitude-longitude coordinate, special treatments are required. In the present study, we used a control volume extension over the polar region with a polar mixing. Figure 1 (a) illustrates the extension of the control volume for the meridional advection over the South Pole, where a polar cell $\left(j=0\right.$ or $\left.j_{\max }+1\right)$ is added to the Gaussian grid $(j=$ $\left.1,2, \ldots, j_{\max }\right)$. This extension is important for the conservative transport with space splitting method. In the splitting procedure, using interface value $f_{\lambda j}\left(j=1,2, \ldots, j_{\max }+1\right)$ and cell-integrated value $q_{\lambda j}\left(j=1,2, \ldots, j_{\max }\right)$ at longitude $\lambda$, we can update $q_{\lambda j}, f_{\lambda j}$ and get the interface fluxes $F_{\lambda j}\left(j=1,2, \ldots, j_{\max }+1\right)$. When the meridional advection is completed for all $\lambda$, the polar cell is predicted according to the finite-volume definition,

$$
q_{\lambda 0}^{t+1}=q_{\lambda 0}^{t}+\frac{F_{\lambda 0}-F_{\lambda 1}}{\Delta}=q_{\lambda 0}^{t}-\frac{F_{\lambda+\pi 0}+F_{\lambda 1}}{\Delta}
$$

for the South Pole; and

$$
\begin{gathered}
q_{\lambda j_{\max }+1}^{t+1}=q_{\lambda j_{\max }+1}^{t}+\frac{F_{\lambda j_{\max }+1}-F_{\lambda j_{\max }+2}}{\Delta} \\
=q_{\lambda j_{\max }+1}^{t}+\frac{F_{\lambda+\pi j_{\max }+1}+F_{\lambda j_{\max }+1}}{\Delta}
\end{gathered}
$$

for the North Pole, where $\Delta$ is grid spacing on the pole. $F_{\lambda 0}=-F_{\lambda+\pi 0}$ and $F_{\lambda j_{\max }+2}=-F_{\lambda+\pi j_{\max }+1}$ are used in the derivations.
The polar mixing technique, which is similar to Ref. (11), is used with the splitting procedure. Considering the fact that the grid points get crowded near the polar region, it is reasonable to re-define the values for each grid points in the polar region with their spatial average. The polar mixing can be given as

$$
q_{i j}=\frac{1}{I_{\max }} \sum_{i=1}^{I_{\max }} q_{i j} \quad j=0,1, J_{\max }, J_{\max }+1,
$$

where $I_{\max }$ is the longitudinal gridpoint number, and $j=$ 0 and $J_{\max }+1$ represents the South Pole and North Pole, respectively. Both the cell-integrated and interface values are redistributed by the polar mixing.

To evaluate the advection in polar region, a "solid bell" transport of Williamson test case $1^{(15)}$ is executed based on this grid system. A steady wind field is specified to be divergence-free as,

$$
\left\{\begin{array}{l}
u=u_{0}(\cos \phi \cos \alpha+\sin \phi \sin \lambda \sin \alpha), \\
v=-u_{0} \sin \lambda \sin \alpha,
\end{array}\right.
$$

where $u_{0}=2 \pi a /(12$ days $)$ and $a$ is the radius of the earth. $\varphi$ and $\lambda$ denotes latitude and longitude respectively. $\alpha$ is a free factor to adjust the wind direction, e.g. $\alpha=0$ noting zonal and $\alpha=\pi / 2$ being meridional current. A cosine bell is initially defined as

$$
q(\lambda, \phi)=\left\{\begin{array}{cc}
\frac{q_{0}}{2}[1+\cos (\pi r / R)] & r<R=a / 3, \\
0 & r \geq R=a / 3,
\end{array}\right.
$$

where $r$ is the distance from the bell center and defined as

$$
r=a \arccos \left[\sin \phi_{c} \sin \phi+\cos \phi_{c} \cos \phi \cos \left(\lambda-\lambda_{c}\right)\right] .
$$

Numerical results show that the CIP-CSLR exactly conserved the cell-integrated average of the transported quantity. Figure 1(b) shows the distributions of the tracer at 

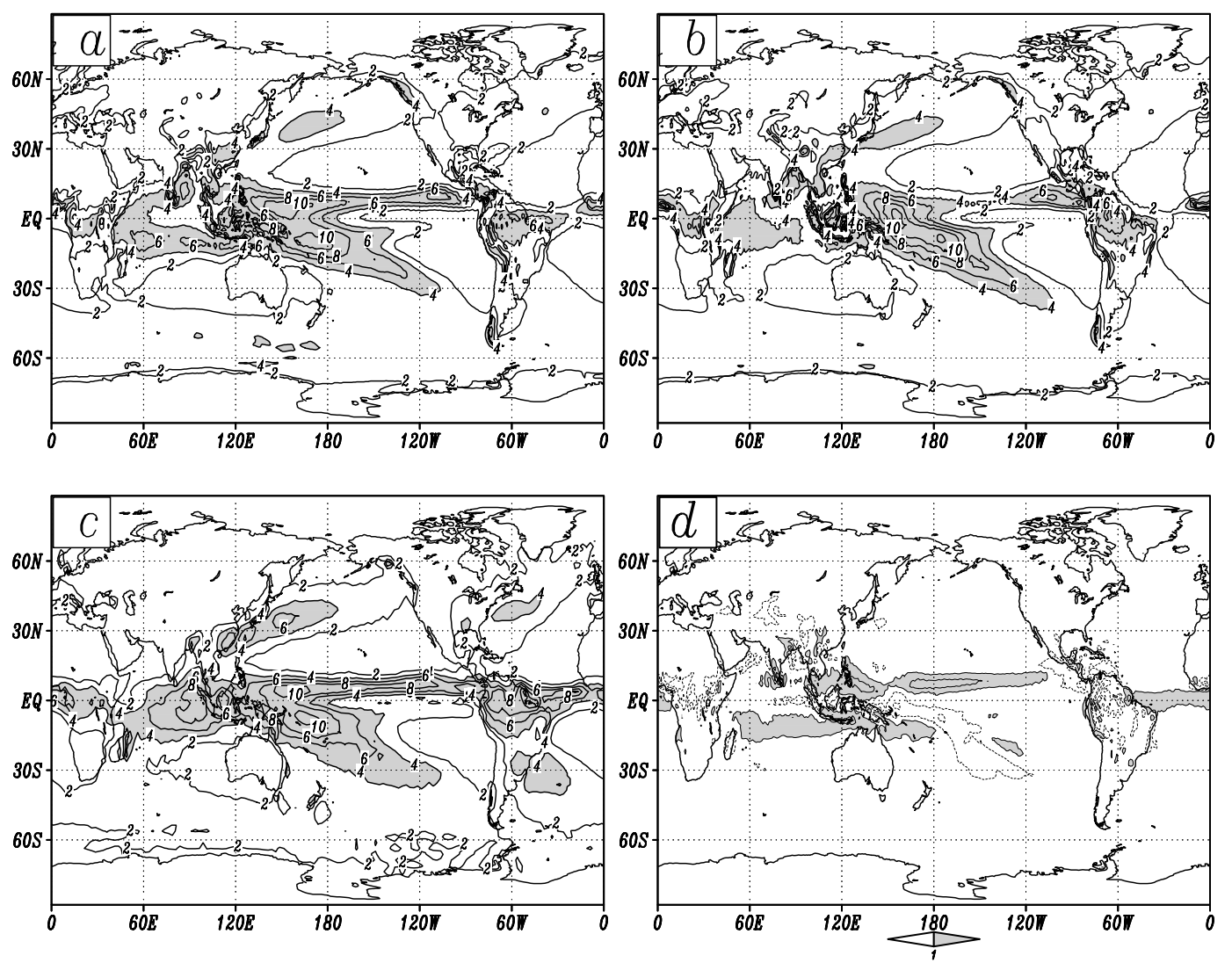

Fig. 2 March-May average precipitation of 20-year T106L20 model runs with the (a) CIPCSLR and (b) spectral method, in comparison with (c) the CMAP analysis. (d) shows (a) - (b).

different instants in the course of moving over the polar region. In spite of the polar singularity in latitude-longitude coordinate, we still get numerical solutions to be comparable to other existing schemes and of adequate accuracy for practical simulations.

As real climatic simulations, numerical experiments over 20-year with the T106L20 (equivalent to 1.125degree horizontal resolution and 20 levels) AFES model including the full physics were carried out on 10 nodes (80 processors) of the Earth Simulator. The AFES is a complex system including many physical processes, e.g. subgrid-scale cumulus convection. The ArakawaSchubert convective scheme ${ }^{(16)}$, which has been widely accepted as one of the most reliable schemes, was chosen as the cumulus convective parameterization in the numerical experiments reported in this paper. We should note that the numerical results of precipitation may depend on physical parameterization schemes. Since our main purpose here is just to illustrate the impact of advection scheme on model output, numerical simulations were carried out with different advection schemes, while the rest part of the model remained the same.

National Centers for Environmental Prediction, National Center for Atmosphere Research (NCEP/NCAR) reanalysis data $\left(2.5^{\circ} \times 2.5^{\circ}\right.$ resolution $)$ is interpolated to the Gaussian transform grid points, and used as the initial condition.

Only the watervapor and liquid water were transported with the CIP-CSLR method in the present study. An inter-comparison between the spectral method and the CIP-CSLR method is shown in Figs. $2-5$ by listing the 20 -year average of the seasonal precipitation. The enhanced CPC Merged Analysis of Precipitation (CMAP) ${ }^{(17)}$ during 1979 and 2002 is taken as the reference to evaluate the numerical results. Though only the watervapor and liquid water are directly computed by the CIP-CSLR, the rainfall is largely improved. Rainband in the InterTropical Convergence Zone (ITCZ) illustrates realistic position and intensity in all four seasons by the new scheme. The original spectral method, however, produces much weaker precipitation in those regions in comparison with the analysis. The rainfall difference (panel $d$ ) between the two schemes shows clearly an under-estimation of condensation in the strong convergence zone in the case of spectral method. Large gradient is hard to be well resolved by the spectral method. The well-distributed ITCZ rainband by the CIP-CSLR is mainly due to the more reliable numerical transport of the water substances.

We also carried out two T319L24 (truncated at wave number 319 with $960 \times 480$ horizontal grids and 24 lev- 

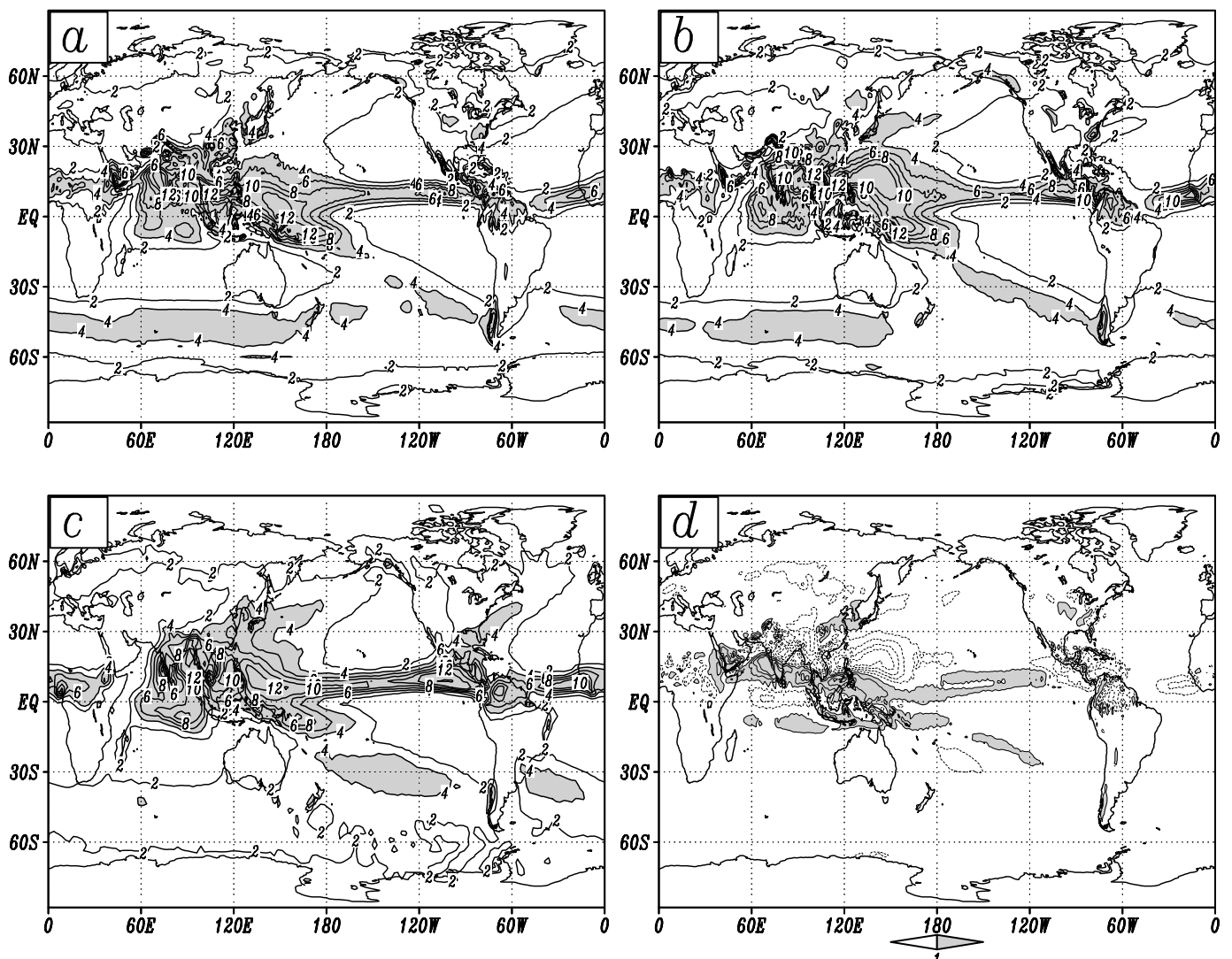

Fig. 3 The same as Fig. 3, but for June-August
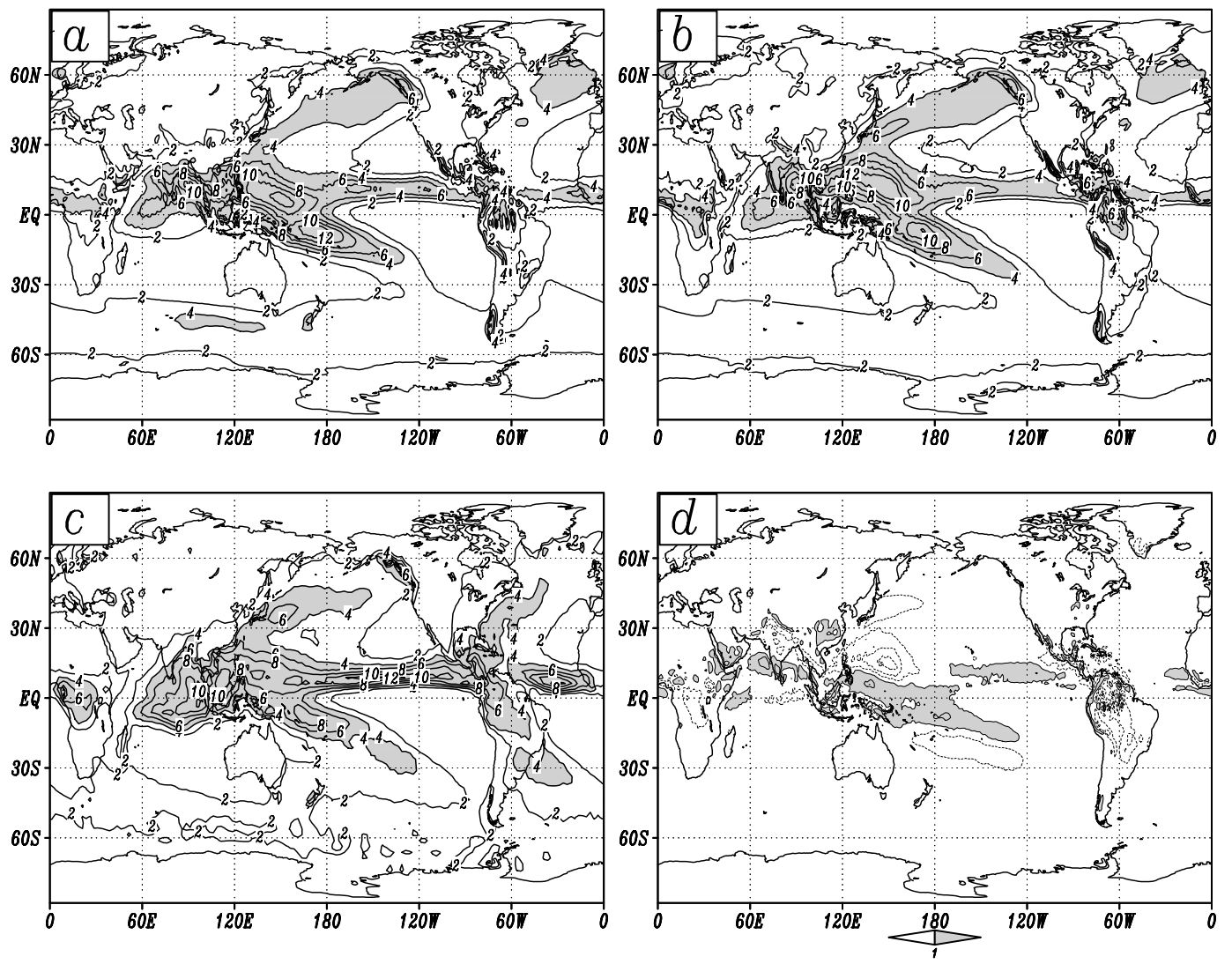

Fig. 4 The same as Fig. 3, but for September-November 

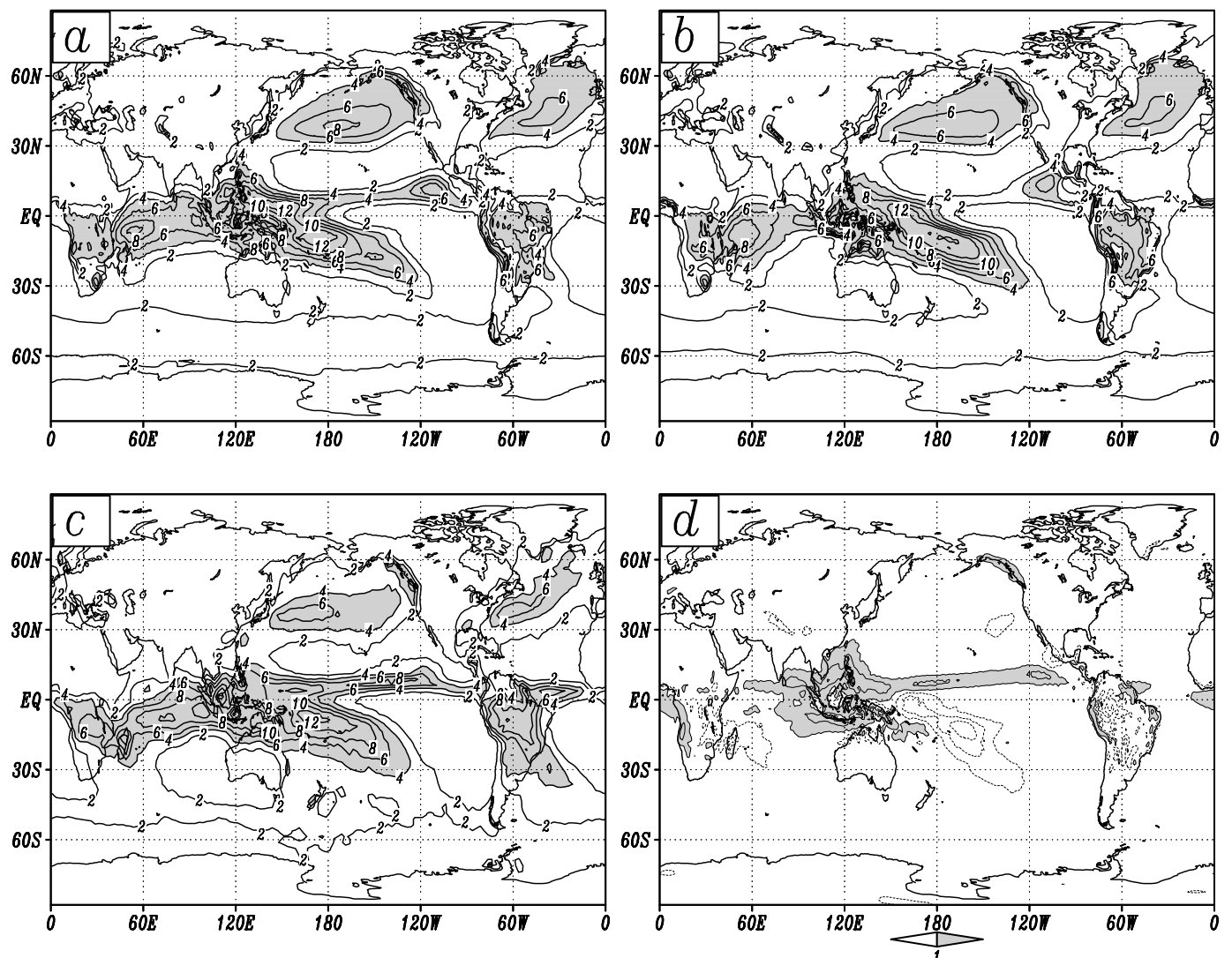

Fig. 5 The same as Fig. 3, but for December-February
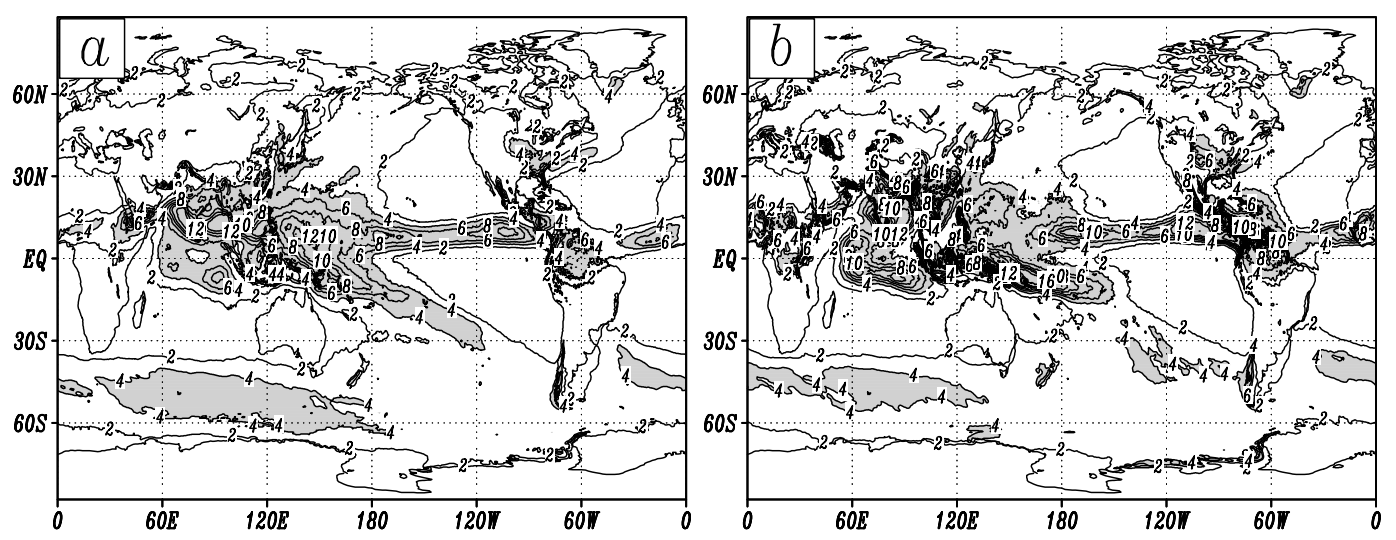

Fig. 6 June-August average precipitation rate simulated during 4-year runs of T319L24 model with (a) the CIP-CSLR and (b) the spectral method. The contours are plotted of 2, 4, 6, $8,10,12,16 \mathrm{~mm} /$ day.

els) model runs under the same model configuration as the previous ones. Figure 6 displays the average precipitation rate during June-August in 4-year integrations. In both the experiments, rainfall distribution in the ITCZ and South Pacific Convergence Zone near Australia is improved evidently in comparison with the low-resolution results in Fig. 3. Figure 6(a) looks much more consistent with the analysis data in Fig. 3 (c). It is clear that the improvement in Fig. 6 (b) is more obvious at equator, and a similar continuous ITCZ rainband as that of the CIP-CSLR scheme can also be produced by the spectral method with finer resolution. However, too much local extreme is observed in Fig. 6 (b). The rainfall pattern by the CIP-CSLR is not changed so much by the grid refinement. This is due to the fact that the CIP-CSLR is able to resolve more subgridscale information by using more than one "moment", i.e. the cell-integrated average and the interface value. Actually, the T106L20 simulation with CIP-CSLR produced adequately realistic output. Therefore, the CIP-CSLR is a more reliable advection scheme for climate simulation. 
Area-weighted zonal mean cloudcover shows great improvement in polar region in the 20-year T106L20 runs (not shown). The idealized solid-bell advection with spectral method also displays more vapor convergence in polar area (Fig. 7), which implies the problem of spectral method in dealing with polar advection base on ArakawaA grid. Though the stability issue is not as serious as the finite difference method in a spectral model, it can not deal with the vapor conservatively over poles on Gaussian (Arakawa-A) grid. In addition, the transformation of watervapor advection between Gaussian grid and spectral space introduces numerical error every timestep. In polar area, excessive water vapor and condensation were observed in the numerical results of the spectral method, which in turn resulted in unrealistic cloudcover in polar region and largely affect the radiative heating in the model. In southern hemisphere, there is plenty of sunshine during December to February. More cloudiness induces higher atmospheric temperature by absorbing solar radiation; and therefore less surface energy income is induced. This is

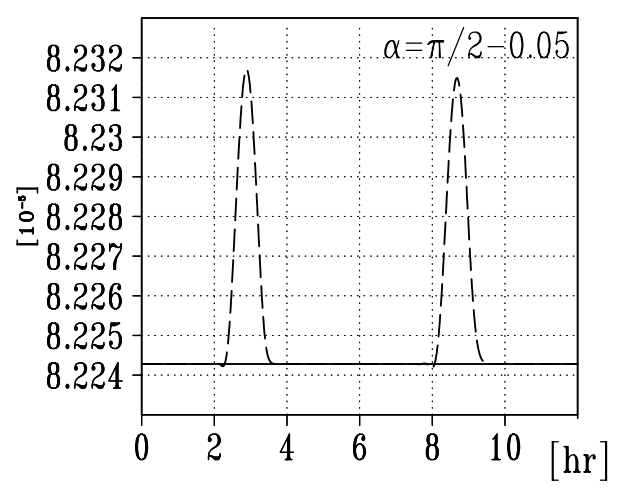

Fig. 7 Time series of global mass integration in a cose-bell advection test with $\alpha=\pi / 2-0.05$. The dashed line shows the original spectral method. And the solid line is the CIP-CSLR. also confirmed by the model output of short- and longwave radiative heating (not shown).

\section{High-Efficiency Global Advection on Yin-Yang Grid}

Even though the polar mixing to some extent overcomes the difficulty in dealing with polar singularity, dissipation error, deformation and the rigorous restriction of timestep under a specified Courant number still remain problems for efficient transport. An effective attempt is to reduce the gridpoint number in polar region. For this purpose, the reduced grid ${ }^{(18)}$, cubic grid ${ }^{(19)}$ and pentagonal/hexagonal grid ${ }^{(20)}$ have been used in various AGCMs. The reduced grid introduces boundaries in the inner area, while the cubic grid possesses eight singular points. The pentagonal/hexagonal grid, on the other hand, is not an orthogonal system, and possesses also singular points. An alternative grid system, which is named the Yin-Yang grid $^{(21)}$, is used here for discretization and evaluation of the CIP-CSLR scheme. It is orthogonal and quasi-uniform grid $^{(22)}$. No singular point exists in the Yin-Yang grid.

Just as shown in Fig. 8 (a), the Yin-Yang system is a composite of two identical grids in spherical geometry that only cover a part of latitudinal region of $[-\pi / 4, \pi / 4]$ and longitudinal region of $[\pi / 4,7 \pi / 4]$. One of them is rotated by $\pi / 2$, which is called the Yin- or E-system. Another one is called Yang- or N-system with no rotating. The minimum grid spacing is about 0.707 times of the maximum one. The grid structure is just the same as the latitudelongitude system and therefore is easy to apply to general circulation model.

In comparison with the reduced grid, the cubic grid or the pentagonal/hexagonal grid, the demerit of YinYang system is the overlapping of the two component grids. Interpolation between the Yin- and Yang-system over boundaries is necessary for the global transport. In
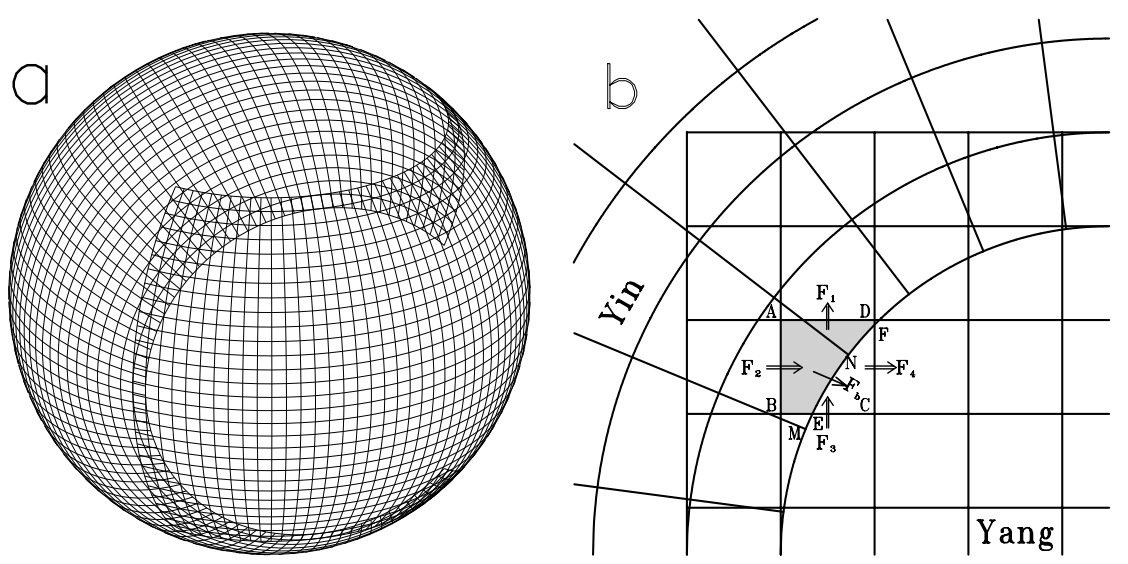

Fig. 8 (a) Schema of Yin-Yang grid on spherical geometry. (b) Conservative interpolation between the Yin and Yang grids. $F_{1,2,3,4}$ shows fluxes trough the four interfaces of a Yang cell, respectively. Curve EF is Yin's boundary located within the Yang cell. $F_{b}$ shows fluxes through EF. 

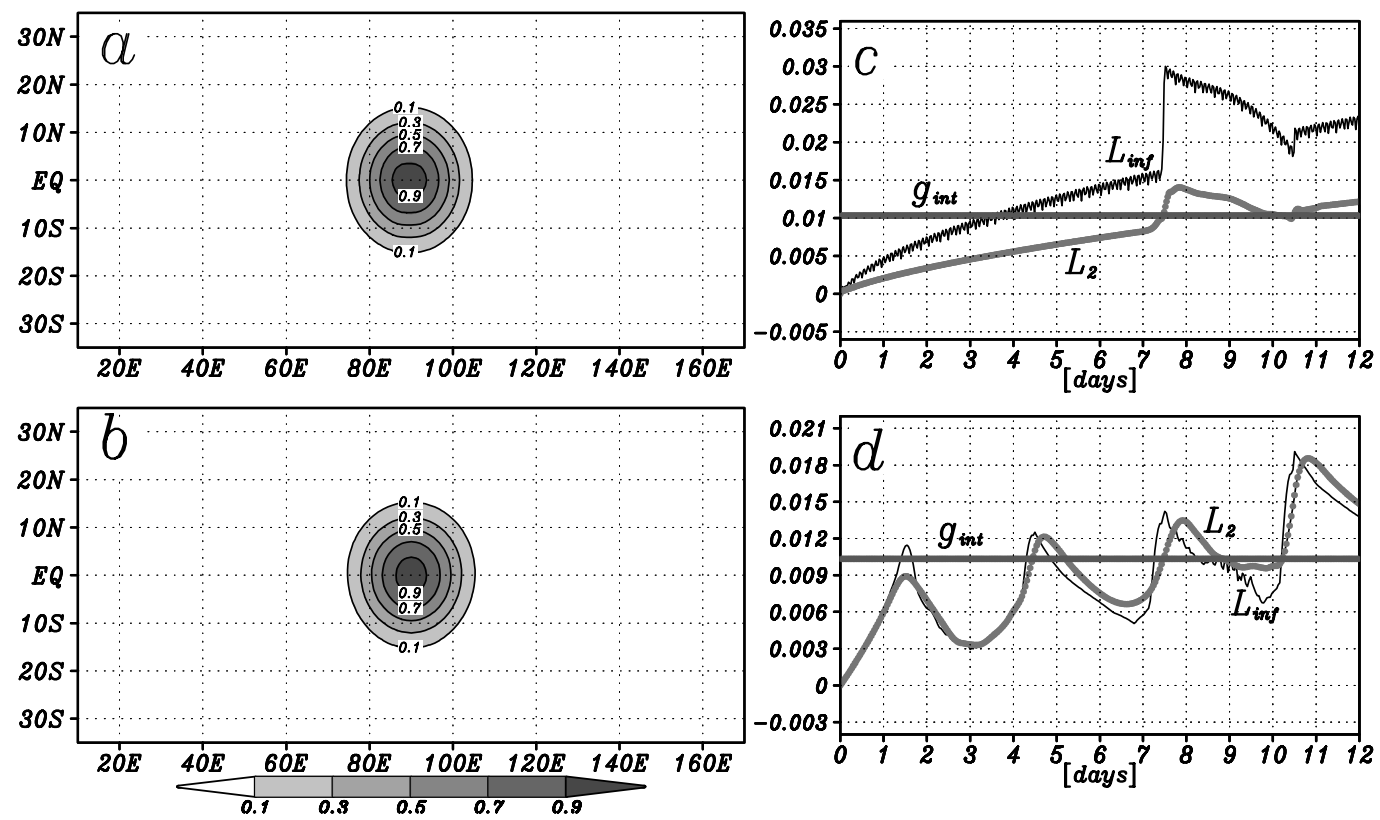

Fig. 9 "Solid bell" advection in one revolution for (a) $\alpha=0$ and (b) alpha $=\pi / 2$. Left panels show numerical results (contour) and the truth (shaded); right ones show $L_{2}$ (filled circle) and $L_{\infty}$ (open circle) norms and global integration of $q\left(g_{\text {int }}\right)$ as a time series.

this study, linear interpolation is conducted on the boundary between the Yin and Yang grids. In order to achieve global conservation, a new scheme for boundary-flux interpolation is suggested. In the overlapping grid, conservation is ensured by constraining the fluxes on each component grid being identical at boundary. As shown in Fig. 8 (b), the flux through boundary EF of the Yin system $\left(F_{b}\right)$ is calculated based the shaded area, which is a part of the inner cell ABCD in the Yang system. The cell ABCD is predicted with the CIP-CSLR splitting procedure independent of the boundary values. Therefore, $F_{1}, F_{2}, F_{3}$, $F_{4}$ and the increment of $q$ within one timestep $(\Delta q)$ are known.

$$
F_{b}=\frac{B E}{B C} F_{3}-F_{1}+F_{2}-\frac{F D}{C D} F_{4}-\Delta q \Delta s
$$

is then derived, where $\Delta s$ represents the shaded area. The flux through cell boundary MN in Yin system is redistributed linearly after $F_{b}$ within all Yang cells being calculated.

We tested the CIP-CSLR with Williamson's "solid bell" advection test ${ }^{(15)}$ on the Yin-Yang grid. The cosine bell is initially located at $90 \mathrm{E}$ on equator. In cases of $\alpha=\pi / 2$ and 0 , the $L_{2}=\left[\Sigma\left(q-q_{\text {true }}\right)^{2} / \Sigma q_{\text {true }}^{2}\right]^{1 / 2}$ and $L_{\infty}=\max \left|q-q_{\text {true }}\right| / \max \left|q_{\text {true }}\right|$ norms and the global integration of $q\left(g_{\text {int }}\right)$ are calculated during one revolution of the transport. Figure 9 displays the horizontal distribution after one-revolution transport and the errors in $L_{2}$ and $L_{\infty}$ series under the resolution of 2.8 degree. The total number of grid points is $96 \times 32 \times 2$. The largest CFL $=1$ is specified through the computation. Shown in Fig. 9, with the conservative adjustment of the fluxes between two grids,

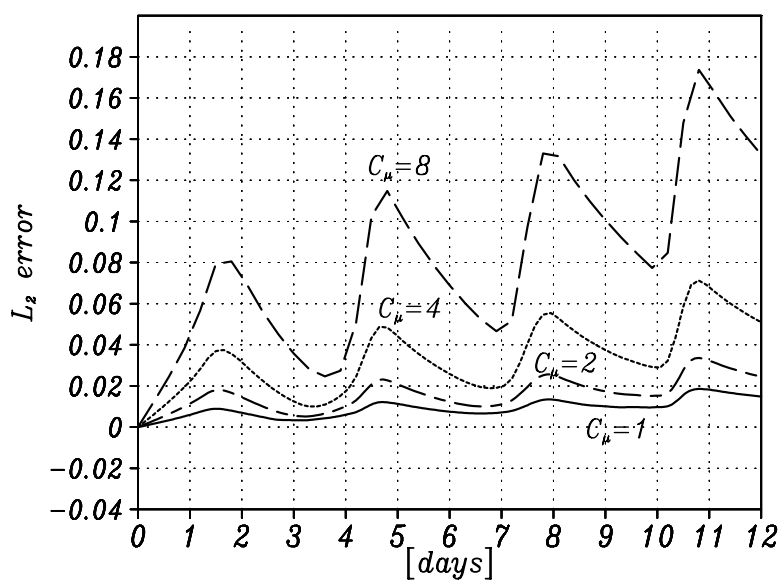

Fig. $10 L_{2}$ norms corresponding for various Courant number in the "Solid bell" test with a resolution of 1.125 degree.

the exact conservation of the transported quantity has been achieved. This makes the Chimera grid quite promising in long term simulation and climatic modeling. Without the polar singularity, the Yin-Yang system can produce much more accurate numerical advection in either the zonal direction or the meridional direction even with fewer grid points. The $L_{2}$ error is only $1 / 20$ of that in latitude-longitude coordinate. Due to the oscillation-free and less diffusive feature of the CIP-CSLR scheme, the numerical solution is positive and shape is well preserved.

In addition, being essentially of a semi-Lagrangian type, the CIP-CSLR remains computationally stable even with a large CFL number. So, efficient computation for advection can be expected in any coordinate though this 
is even more meaningful in the latitude-longitude coordinate. Figure 10 gives the $L_{2}$ errors for various Courant numbers in case of $\alpha=\pi / 2$ with a resolution of $1.125 \mathrm{de}$ gree. It is equivalent to the resolution of T106 spectral discretization. Because the computation of the trajectory in the numerical experiments is of the first order, a large time step tends to cause larger numerical error even the numerical diffusion is reduced. This can be improved by using higher order trajectory scheme. Thus, the $L_{2}$ error increases with Courant number and time, nevertheless, it is still acceptable for global advection with the meridional Courant number up to 4 in this resolution.

\section{Summary}

Advection scheme CIP-CSLR has been implemented and tested in an atmospheric general circulation model which is built on a latitude-longitude grid and a new overlapping grid system. Amelioration of the model representation by the CIP-CSLR is verified by both idealized tests and long-term climatic simulations. Numerical experiments show that CIP-CSLR is capable of transporting water substances more accurately in comparison with the spectral method which appears numerically oscillatory. Realistic rainfall is achieved by using the robust advection scheme. In addition, atmospheric thermodynamic structure is also improved largely, especially in equatorial and high-latitude region.

Based on the overlapping Yin-Yang grid system, the CIP-CSLR gives accurate numerical solutions to the advection by dimensional splitting. With special treatments over the boundary cells, the exact conservation of the transported quantity is obtained. Implementing CIPCSLR on the Yin-Yang grid provides a conservative, accurate and efficient framework for numerical advection on spherical geometry, which then promisingly leads to a practical model for the simulations of atmospheric general circulation and climate system. As a practice toward such a direction, we are currently developing a new global nonhydrostatic model at the Earth Simulator Center, where the Yin-Yang grid and CIP-CSLR are adopted for spatial discretization and advection computing.

\section{Acknowledgment}

The authors would like to thank Dr. Kageyama at the Earth Simulator Center for his kind help on the YinYang grid. We appreciate the valuable suggestions of two anonymous reviewers.

\section{References}

( 1 ) Harten, A., High Resolution Schemes for Hyperbolic Conservation Laws, J. Comput. Phys., Vol.49 (1983), pp.357-393.

( 2 ) van Leer, B., Toward the Ultimate Conservative Difference Scheme. V. A Second Order Sequel to Godunov's Method, J. Comput. Phys., Vol.32 (1979), pp.101-136.
( 3 ) Colella, P. and Woodward, P.R., The Piecewise Parabolic Method (PPM) for Gas-Dynamical Simulations, J. Comput. Phys., Vol.54 (1984), pp.174-201.

( 4 ) Yabe, T., Xiao, F. and Utsumi, T., The Constrained Interpolation Profile Method for Multiphase Analysis, J. Comput. Phys., Vol.169 (2001), pp.556-593.

( 5 ) Peng, X., Xiao, F., Yabe, T. and Tani, K., Implementation of the CIP as the Advection Solver in the MM5, Mon. Wea. Rev., Vol.131 (2003), pp.1256-1271.

( 6 ) Xiao, F., Yabe, T., Peng, X. and Kobayashi, H., Conservative and Oscillation-Less Atmospheric Transport Schemes Based on Rational Function, J. Geophys. Res., Vol.107 (D22) (2002), p.4609.

( 7 ) AFES Team, Model Document of the Spectral AGCMAFES1.0, Earth Simulator Developing Report, (in Japanese), Japan, (2002).

( 8 ) Xiao, F., Yabe, T. and Ito, T., Constructing Oscillation Preventing Scheme for Advection Equation by Rational Function, Comput. Phys. Commun., Vol.93 (1996), pp.1-12.

(9) Strang, G., On the Construction and Comparison of Difference Schemes, SIAM J. Numer. Anal., Vol.5 (1968), pp.506-517.

(10) Clappier, A., A Correction Method for Use in Multidimensional Time-Splitting Advection Algorithms: Application to Two-and Three-Dimensional Transport, Mon. Wea. Rev., Vol.126 (1998), pp.232-242.

(11) Hundsdorfer, W. and Spee, E.J., An Efficient Horizontal Advection Scheme for Modeling of Global Transport of Constituents, Mon. Wea. Rev., Vol.123 (1995), pp.3554-3564.

(12) Ritchie, H., Temperton, C., Simmons, A., Hortal, M., Davies, T., Dent, D. and Hamrud, M., Implementation of the Semi-Lagrangian Method in a High-Resolution Version of the ECMWF Forecast Model, Mon. Wea. Rev., Vol.123 (1995), pp.489-514.

(13) Williamson, D.L. and Rasch, P.J., Water Vapor Transport in the NCAR CCM2, Tellus, A, Vol.46 (1994), pp.34-51.

(14) Lin, S.-J. and Rood, R.B., Multidimensional FluxForm Semi-Lagrangian Transport Schemes, Mon. Wea. Rev., Vol.124 (1996), pp.2046-2070.

(15) Williamson, D.L., Drake, J.B., Hack, J.J., Jakob, R. and Swarztrauber, P.N., A Standard Test Set for Numerical Approximations to the Shallow Water Equations in Spherical Geometry, J. Comput. Phys., Vol.102 (1992), pp.211-224.

(16) Moorthi, S. and Suarez, M.J., Relaxed ArakawaSchubert: A Parameterization of Moist Convection for General Circulation Models, Mon. Wea. Rev., Vol.120 (1992), pp.978-1002.

(17) Xie, P. and Arkin, P.A., Analyses of Global Monthly Precipitation Using Gauge Observation, Satellite Estimation, and Numerical Model Prediction, J. Climate, Vol.9 (1996), pp.840-859.

(18) Rasch, P.J., Conservative Shape-Preserving TwoDimensional Transport on a Spherical Reduced Grid, Mon. Wea. Rev., Vol.122 (1994), pp.1337-1350.

(19) McGregor, J.L., Semi-Lagrangian Advection on Conformal-Cubic Grids, Mon. Wea. Rev., Vol.124 
(1996), pp.1311-1322.

(20) Tomita, H., Tsugawa, M., Satoh, M. and Goto, K., Shallow Water Model on a Modified Icosahedral Geodesic Grid by Using Spring Dynamics, J. Comput. Phys., Vol.174 (2001), pp.579-613.

(21) Kageyama, A. and Sato, T., The "Yin-Yang grid": an Overset Grid in Spherical Geometry, Geochem.
Geophys. Geosys., Vol.5 (2004), Q09005, doi: 10.1029/2004GC000734.

(22) Peng, X., Xiao, F. and Takahashi, K., Accurate and Efficient Advection Computing on a Sphere, 17th Symposium on Numerical Fluid Mechanics, Tokyo, Japan, (2003), p.107. 